\title{
Probabilistic assessment for clavicle fracture under compression loading: rate-dependent behavior
}

\author{
D. Sánchez-Molina ${ }^{a}$, S. García-Vilana ${ }^{a}$, J. Velázquez-Ameijide ${ }^{a}$ \\ C. Arregui-Dalmases ${ }^{b}$
}

19 June 2020

${ }^{a}$ UPC, EEBE-GRABI, Eduard Maristany, 14, 08019 Barcelona

${ }^{b}$ Centro Zaragoza, Crtra. 232, km.273, 50690 Pedrola

CITE: Sánchez-Molina, D., García-Vilana, S., Velázquez-Ameijide, J. \& Arregui-Dalmases, C. (2020): Probabilistic assessment for clavicle fracture under compression loading: ratedependent behavior, Biomedical Engineering: Applications, Basis and Communications, 32(5), 2050040. DOI: $10.4015 / \mathrm{S} 1016237220500404$

\begin{abstract}
Pedestrians, motorcyclists, and occupants of vehicle involved in side impacts can suffer lateral impacts at the shoulder with sufficiently high forces to fracture the clavicle. While some previous studies have developed a force tolerance for the clavicle, this study examines the clavicle under compression forces, both under dynamic and quasi-static loading conditions and then the experimentally fracture patterns are compared with those identified in actual road traffic crashes. The quasi-static and dynamic tests were conducted in 22 clavicles from 14 donors aged 14-56 (10 males and 3 females, mean age $44.4 \pm 13.2$ ). Clavicles were tested under compression forces until complete fracture. Probability of fracture is around $50 \%$ for $2335 \mathrm{~N}$ in the quasi-static case (low strain rate) and $3045 \mathrm{~N}$ in the dynamic case (high strain rate). In addition, risk curves for fracture have been computed from the sample. The experiments showed large differences in the peak force both in quasi-static $(2416 \pm 781 \mathrm{~N})$ and dynamic $(3183 \pm 981 \mathrm{~N})$ tests, but the displacement at the peak force was very similar $(4.9 \pm 1.1 \mathrm{~mm}$ in quasi-static and $5.0 \pm 1.3 \mathrm{~mm}$ in dynamic tests). Thus, a stiffer behavior of the specimens was observed for the dynamic tests. The Probability Density Function for dynamic and quasi-static cases differ significantly $(p$-value $<0.04)$. The fracture patterns were consistent with principles of structural mechanics, predicting that the fracture will occur at points near to the highest bending moment point, and with the fractures observed in real road crashes.
\end{abstract}


KEYWORDS: Clavicle, Fracture, Injury Pattern, Probabilistic Risk Curve for clavicle fracture.

\section{Introduction}

The human shoulder is one of the most frequently affected anatomical structures in road crashes such as roll-overs, side crashes, motorcyclists, pedestrian or bicycle crashes. Franmpton and coworkers investigated 5362 road crashes in side impacts, reporting that injuries occurred in the shoulder $50 \%$ of AIS2+, this number increased to $64 \%$ for roll-over traffic accidents [1]. The same study showed that clavicle fracture accounts for approximately $66 \%$ of shoulder injuries in side impact collisions and $97 \%$ in roll-over traffic accidents. A later study reported that clavicle fracture accounts for about $4 \%$ of all fractures, with the middle third of the clavicle being the most common location of the fracture, quantified as $69 \%-82 \%$ [2].

In spite of being a very common fracture, clavicle fracture have not been sufficiently researched and there is only a modest number of studies in the field of impact biomechanics or medical emergencies, the latter being a field where scientific production is growing at a greater rate [3]. The purpose of this study is to evaluate the biomechanical response of the human clavicle subjected to axial compression. This research focuses on the effect of impact velocity on the fracture force, as well as its implication in the fracture location because an improved biomechanical understanding of the clavicle fracture is essential for the validation of finite element models as well as for the estimation of the severity of the situation by emergency professionals.

The medical literature makes explicit the extent to which clavicle fractures are important, for example in [4] it is stated that clavicle fractures are frequent injuries, accounting for about $3 \%$ of all fractures, and are more common in young, active individuals. With regard to the location, fractures of the midshaft are the most common, representing approximately $75 \%$ of all clavicle fractures [5]. In addition, a number of studies have assessed the effectiveness of treatment for clavicle fractures; however, the results are inconsistent and inconclusive because of the small sample size in most of the studies [6], among other factors.

With respect to fracture risk assessment, an important question about the strength of human clavicles and their risk of fracture under major compressive stresses is that many studies are based on conducting quasi-static compression tests and extrapolate from these tests the risk of fracture in traffic collisions. However, as our work suggests, there are significant differences between quasistatic (low strain rate) and dynamic (high strain rate) situations ( $p$-value $<0.04)$. Indeed, these two situations differ so much that it seems that it is not possible to reliably use quasi-static test results in dynamic situations, due to the enormous differences observed in strength, as discussed below. One of the intentions of this paper is to clarify this point, and to suggest that quasi-static trials should 
no longer be used to assess the risk for road traffic accidents and other situations associated with serious injury.

\section{Materials and methods}

\subsection{Materials}

In this study axial compression tests were conducted using 24 clavicles: 14 under quasi-static conditions and 10 under dynamic conditions. The binding and kinematic constrains were controlled to be equivalent to the constrains of a clavicle placed in its natural position inside the shoulder under a lateral impact, in a high energy crash [7].

The 24 clavicles were extracted from 14 donors, 11 men and 3 women, ranging in age from 14 to 56 years, with a mean age and standard deviation of $44.4 \pm 13.2$ years. The clavicles were provided by the Department of Anatomy of the Faculty of Medicine of the University of Navarra, the Pathological Anatomy Service of the Clínica Universitaria de Navarra (CUN) and the Tissue Donation Program of the Hospital Virgen del Camino, Navarra. For all the samples, a Computerized Tomography (CT) was performed for each specimen just after the extraction, to rule out any fracture or significant defect in the cortical area. Once the clavicles were extracted, the soft tissue adjacent to the bone was carefully removed and the clavicles were frozen to $-20^{\circ} \mathrm{C}$ in individual bags. The storage period ranged from one week to 18 months. The day before the experiment, the clavicles were exposed to room temperature, during this thawing phase the clavicles were wrapped in a gauze soaked in saline solution.

\subsection{Experiment}

Using a previous methodology described in the literature [9, 10], both ends of the clavicle were placed in aluminum cup, and then the cups were filled with a self-solidifying material known as Fast-Cast ${ }^{\circledR}$ (Product 891, Goldenwest Mfg., Inc., Cedar Ridge, CA, USA). To guarantee the right alignment of the clavicle, a cylindrical guide was used to ensure relative immobility during the curing process of the self-solidifying material. The clavicle with its ends rigidly joined to the aluminum cups were mechanically fixed to the designed mechanism and adapted to two different testing machines: for the static tests (INSTRON 4502; Norwood, MA, USA) and for the dynamic tests (MTS 819 High Rate Test System, MN, USA). The same axial compression conditions were considered for both tests. The placement of the acromial joint of the clavicle only allowed Zshift and rotation was totally restricted (see Figure 1, for the axis orientation), and in the case of the sternal joint no displacement was allowed, and a controlled and measured rotation on the X-axis was allowed. The $S$-shape of the clavile is aligned with the XZ-plane; regularly the $Z$-axis is aligned with the sternalacromial direction. 


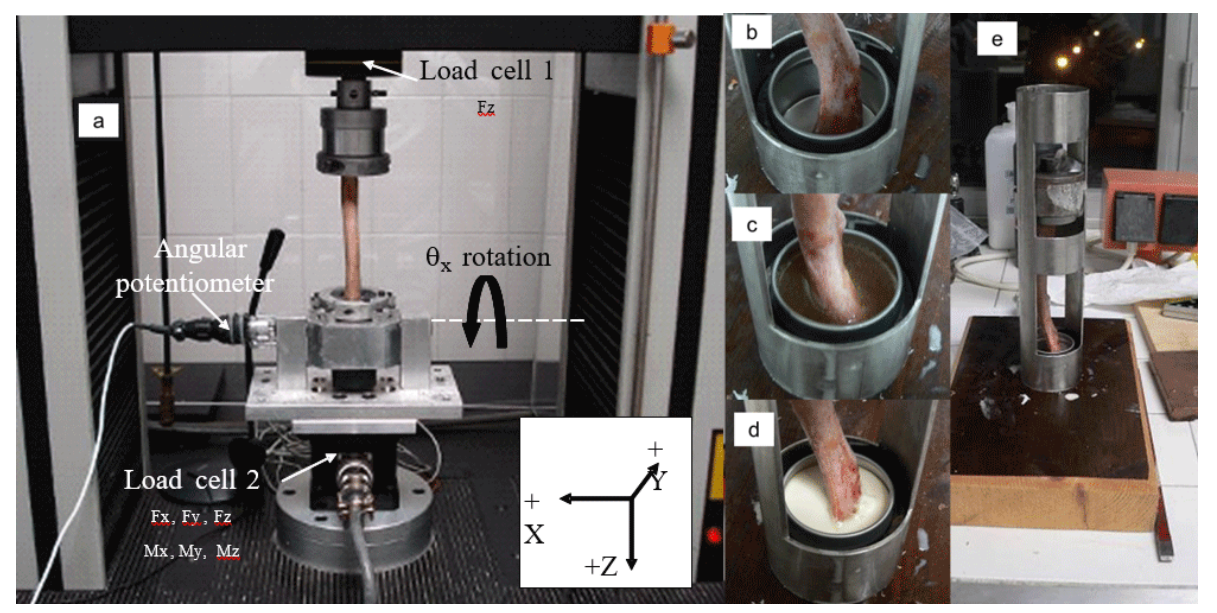

Figure 1: (a) general view of the compression test, (b) pre-filling of the cup with the twocomponent mixture, (c) filling of the two-component mixture, (d) completion of the curing process, (e) general view of the outer cylinder to ensure the alignment of the cups. Z-axis is aligned in the sternal end-acromial end direction, ZY-plane is contained in the main plane of the clavicle.

Each clavicle was mounted vertically, with the acromial extremity at the top and the sternal extremity at the bottom (see Figure 1), always maintaining the same rotation on the $Z$-axis. The instrumentation for the quasi-static tests included a single-axis load cell (INSTRON 2518-804, Norwood, MA, USA) mounted on the acromial extremity of the clavicle, a 6-axis load cell (MC3A6-1000, Advanced Mechanical Technology Inc, Watertown, MA, USA) and an external end mounted angle potentiometer. Data was collected at $2000 \mathrm{~Hz}$ using a National Instruments Model NI cDAQ-9172 data acquisition system (modules NI9237 and NI 9215, National Instruments Corporation, Austin, TX, USA) and filtered according to standard practices. The instrumentation for the dynamic tests included a single-axis load cell (Kistler 9341A, Winterthur, Switzerland), an accelerometer (Brüel \& Kjaer 4384, Nærum, Denmark), and the same 6axis load cell, the same rotational potentiometer and the same data acquisition system. The dynamic data was collected at $5000 \mathrm{~Hz}$ and filtered according to recommended standard practices.

The quasi-static tests were performed at a speed of $0.63 \mathrm{~mm} / \mathrm{s}$ and the dynamic tests at $1000 \mathrm{~mm} / \mathrm{s}$. These speeds correspond to strain rates of approximately $\dot{\varepsilon}=0.004 \mathrm{~s}^{-1}$ and $\dot{\varepsilon}=6.70 \mathrm{~s}^{-1}$. The tests were documented through digital photographs and video camera at 1000 frames per second. The forces were measured in the sternal extremity of the clavicle. The fracture peak was defined as the maximum $F_{z}$ value and the fracture force as the magnitude of $F_{z}$ immediately prior to the first abrupt descent in the quasi-static tests and the largest descent in the dynamic tests. 


\subsection{Logistic regression and Fracture probability curves}

A risk curve is a curve defined as the graph of an increasing monotonic function which assigns a probability of fracture $0<p_{F}<1$ to a value $F$ of the compression force. A logistic regression procedure has been used to compute the best risk curve modeling the experimental data. Logistic regression is the adequate regression analysis to perform when the dependent variable is dichotomous (it takes only two values). In this study, the logistic regression is intended to be a predictive analysis and this case, the two possible value are "observed fracture" (1) or "non-observed fracture" (0). Thus, logistic regression is used to describe the probability of fracture $0 \leq p_{F} \leq 1$ of fracture.

For both the dynamic tests and the both quasi-static tests, an ordered sample with fracture values $F_{1}<F_{2}<\cdots<F_{n}$ was obtained. The logistic regression aims to approximate the probability $p_{F}$ that a certain value of force $F$ involves bone fracture, as shown in the following equation:

$$
p_{F}=G(F)=\frac{1}{1+\exp (\alpha+\beta F)}
$$

where the coefficients $\alpha$ and $\beta$ can be obtained by linear regression. Since the function in the second member meets the requirements to be a probability distribution function and is also invertible, this relationship can be written as follows:

$$
\ln \left(\frac{1-p_{F}}{p_{F}}\right)=\alpha+\beta F
$$

For the estimation of $\alpha$ and $\beta$ we construct a sample of the form $\left(F_{i}, \ln [(1-\right.$ $\left.\left.\left.p_{F, i}\right) / p_{F, i}\right]\right)$, where for a sample size $n$, the probabilities obtained from the empirical distribution function on the sample values are $p_{F, i}=i / n$. By means of equation (2) and a linear regression procedure, the best-fit parameters $\alpha$ and $\beta$ can be obtained.

\section{Results}

\subsection{Force-Displacement Curves}

In all the 24 tests, clavicles were brought to fracture. The force is always measured at the acromial extremity and is defined as the compression force $\left(F_{z}\right)$. In addition, the movement of the sternal extremity was measured by means of an extensometer, being the only degree of freedom in the system (table 1).

The slope of force-displacement curves is considerably different in quasistatic and dynamic curves. In quasi-static tests, a linear behavior is observed between 1 and $4 \mathrm{~mm}$, preceded by a smooth descent (in most cases), and finally an abrupt descent of the force, which is defined as the point of fracture. The peak varied significantly between $1207 \mathrm{~N}$ (Test 10L) and $3665 \mathrm{~N}$ (Test 15R). Interestingly, there doesn't seem to be a relationship between peak force and 
Table 1: Force and displacement results for quasi-static and dynamic tests.

\begin{tabular}{|c|c|c|c|c|c|c|c|c|c|}
\hline Donor & Gender & $\begin{array}{c}\text { Age } \\
\text { [years] }\end{array}$ & Side & $\begin{array}{c}\text { Loading } \\
\text { Type }\end{array}$ & $\begin{array}{c}\text { Peak } \\
\text { Force } \\
{[\mathrm{N}]}\end{array}$ & $\begin{array}{c}\text { Peak } \\
\text { Displacement } \\
{[\mathrm{mm}]}\end{array}$ & $\begin{array}{c}\text { Fracture } \\
\text { Force } \\
{[\mathrm{N}]}\end{array}$ & $\begin{array}{c}\text { Fracture } \\
\text { Displacement } \\
{[\mathrm{mm}]}\end{array}$ & $\begin{array}{c}\text { Elongation } \\
(\%)\end{array}$ \\
\hline 1 & $\mathrm{M}$ & 47 & $\mathrm{R}$ & Quasi-static & 2478 & 4.0 & 2475 & 3.2 & 2.2 \\
\hline 3 & M & 49 & $\mathrm{~L}$ & Quasi-static & 2692 & 5.1 & 1362 & 9.5 & 5.8 \\
\hline 4 & M & 54 & $\mathrm{~L}$ & Quasi-static & 3324 & 6.6 & 3135 & 6.7 & 4.0 \\
\hline 5 & M & 55 & $\mathrm{~L}$ & Quasi-static & 2235 & 5.1 & 2067 & 6.8 & 4.0 \\
\hline 6 & M & 56 & L & Quasi-static & 3310 & 4.0 & 3310 & 4.0 & 2.5 \\
\hline 7 & M & 56 & $\mathrm{~L}$ & Quasi-static & 2167 & 6.8 & 2057 & 7.6 & 5.1 \\
\hline 8 & M & 23 & $\mathrm{R}$ & Quasi-static & 2966 & 4.6 & 1495 & 10.5 & 7.2 \\
\hline 9 & M & 37 & $\mathrm{R}$ & Quasi-static & 1672 & 5.1 & 1308 & 12.4 & 7.7 \\
\hline 10 & $\mathrm{~F}$ & 14 & $\mathrm{~L}$ & Quasi-static & 1207 & 3.2 & 624 & 9.1 & 6.9 \\
\hline 11 & $\mathrm{~F}$ & 43 & $\mathrm{R}$ & Quasi-static & 2359 & 4.6 & 1016 & 12.3 & 8.2 \\
\hline 12 & M & 46 & $\mathrm{R}$ & Quasi-static & 2178 & 6.4 & 1926 & 12.4 & 8.3 \\
\hline 13 & $\mathrm{~F}$ & 46 & $\mathrm{~L}$ & Quasi-static & 1570 & 3.7 & 1165 & 8.2 & 5.6 \\
\hline 14 & M & 42 & $\mathrm{R}$ & Quasi-static & 1878 & 4.5 & 1729 & 6.0 & 3.7 \\
\hline \multirow[t]{3}{*}{15} & M & 56 & $\mathrm{R}$ & Quasi-static & 3665 & 4.7 & 3110 & 7.4 & 4.7 \\
\hline & & & & Mean & 2407 & 4.9 & 1912 & 8.3 & 5.42 \\
\hline & & & & Std.Dev. & 720.1 & 1.1 & 838.6 & 2.9 & 2.02 \\
\hline \multirow[t]{2}{*}{ Donor } & Gender & Age & Side & Loading & Peak & Peak & Fracture & Fracture & Elongation \\
\hline & & [years] & & Type & $\begin{array}{l}\text { Force } \\
{[\mathrm{N}]}\end{array}$ & $\begin{array}{c}\text { Displacement } \\
{[\mathrm{mm}]}\end{array}$ & $\begin{array}{l}\text { Force } \\
{[\mathrm{N}]}\end{array}$ & $\begin{array}{l}\text { Displacement } \\
{[\mathrm{mm}]}\end{array}$ & \\
\hline 5 & $\mathrm{M}$ & 55 & $\mathrm{R}$ & Dynamic & 2967 & 3.5 & 2358 & 5.4 & 3.2 \\
\hline 6 & M & 56 & $\mathrm{R}$ & Dynamic & 4318 & 4.2 & 3841 & 4.3 & 2.8 \\
\hline 7 & M & 56 & $\mathrm{R}$ & Dynamic & 5011 & 5.3 & 4097 & 7.6 & 5.1 \\
\hline 9 & M & 37 & $\mathrm{~L}$ & Dynamic & 2578 & 4.2 & 1919 & 6.3 & 3.8 \\
\hline 10 & $\mathrm{~F}$ & 14 & $\mathrm{R}$ & Dynamic & 1959 & 7.6 & 1607 & 9.5 & 7.3 \\
\hline 11 & F & 43 & $\mathrm{~L}$ & Dynamic & 2716 & 5.1 & 2010 & 7.3 & 4.9 \\
\hline 12 & M & 46 & $\mathrm{~L}$ & Dynamic & 2980 & 5.2 & 2906 & 5.4 & 3.5 \\
\hline 13 & F & 46 & $\mathrm{R}$ & Dynamic & 2645 & 6.1 & 2376 & 7.0 & 5.0 \\
\hline 14 & M & 42 & $\mathrm{~L}$ & Dynamic & 2453 & 5.4 & 1722 & 10.0 & 5.9 \\
\hline \multirow[t]{3}{*}{15} & M & 56 & $\mathrm{~L}$ & Dynamic & 4199 & 3.2 & 3650 & 4.8 & 3.0 \\
\hline & & & & Mean & 3182 & 5.0 & 2648 & 6.7 & 4.45 \\
\hline & & & & Std.Dev. & 980.6 & 1.3 & 920.8 & 1.9 & 1.46 \\
\hline
\end{tabular}

fracture force, with the post-peak varying between specimens. Fracture strength varied between $43 \%$ and $100 \%$ of the peak force, with its magnitude range between $624 \mathrm{~N}$ (TEST 10L) and $3.3 \mathrm{kN}$ (TEST 06L). Figure 2(a) reproduces the force-displacement curves for the quasi-static tests.

The curves obtained for the dynamic tests presented a very different aspect from the quasi-static ones, varying the linear component from one curve to another. In the initial phase loading process, it appears a higher slope than for the quasi-static tests, and the curves present a more wavy shape, mainly due to the speed of the load application. Peaks in force vary between $1959 \mathrm{~N}$ (Test $10 \mathrm{R}$ ) and $5010 \mathrm{~N}$ (Test 07R), with these peaks being considerably lower than in quasi-static tests. Finally, the range of force at fracture varies between 1607 N and 4097 N. Figure 2(b) reproduces the Force-Displacement Curves for the dynamic tests.

\subsection{Risk Curves for Fracture}

The logistic regression for the dynamic and quasi-static tests is presented in Figure 3(a). In both cases a good fit is observed and it is seen that on average, 

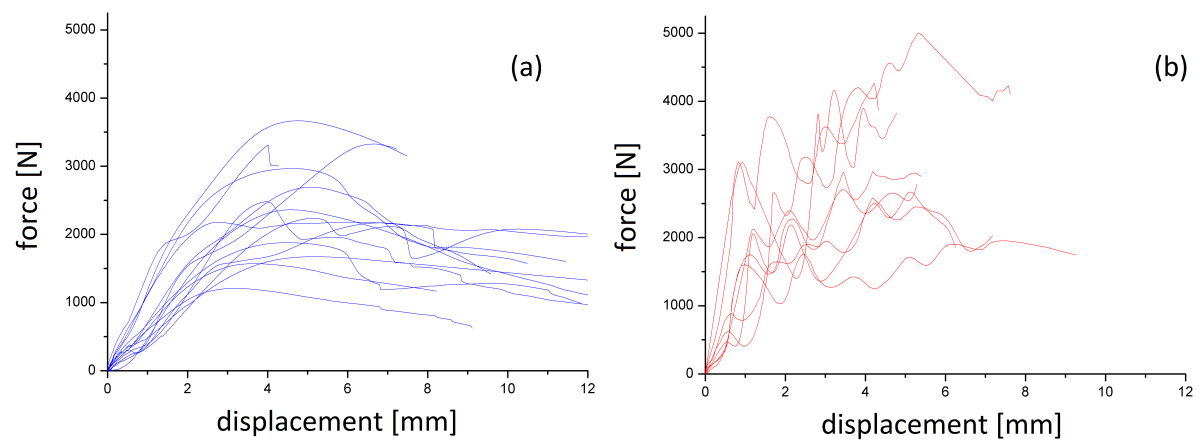

Figure 2: Force-displacement curves for (a) quasi-static tests and (b) dynamic tests. A wavy behavior is observed for curves for the dynamic tests and it is conjectured that this behavior is associated with the propagation of elastic waves and due to imperfectly-balanced vibrations along the specimen. This contrasts with smoother increasing-decreasing behavior of the curves for quasi-static tests.

the fracture values for the dynamic tests is higher than for the quasi-static tests.

The calculated regression parameters are as follows: for dynamic tests $\alpha=$ 4.8701 and $\beta=-0.0016\left(r^{2}=0.8647, p<0.001\right)$ and for quasi-static tests $\alpha=$ 5.1399 y $\beta=-0.0022\left(r^{2}=0.9788, p<0.001\right)$. From these values, the probability densities can be obtained, and once integrating the probability density function, the probability curves of accumulated fracture can be represented, which allows us to assign a probability of fracture to each value of the axial force (Figure $3(\mathrm{~b}))$.
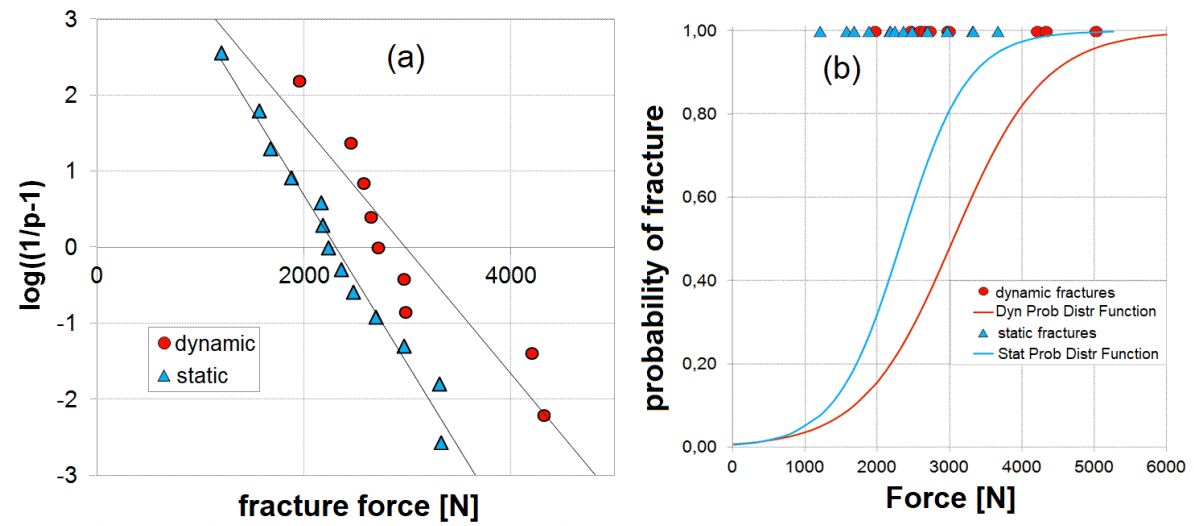

Figure 3: (a) Linear regressions for fracture values and (b) Probability Distribution Functions for dynamic and quasi-static tests. 


\subsection{Localization of fractures}

With respect to the location of fracture, most fractures (about 70\%) were located in the middle third. This observation is consistent with the scientific literature for severe road crashes. With respect to type, about $60 \%$ of all fractures have a butterfly-wing shape. In Figure 4 the location of fractures for quasi-static and dynamic tests is shown. The most frequent type is shown in Figure 5: a butterfly-wing shape in which the start of the crack is in the opposite side of maximum compression stress, near the most outer point of the clavicle arch.

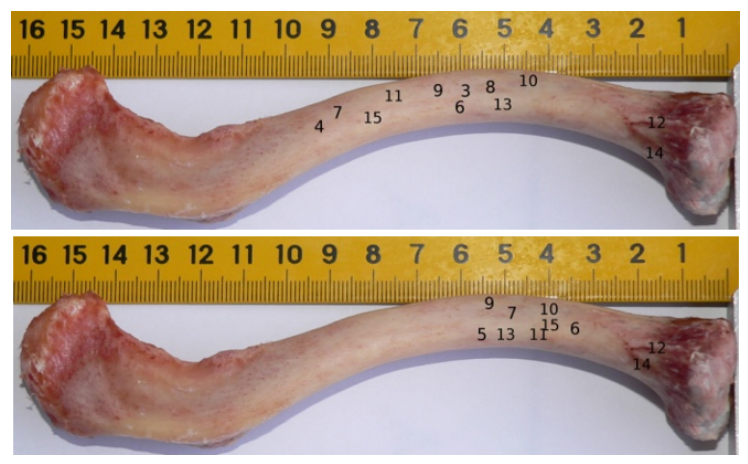

Figure 4: Location of fractures, the numbers represent the numbers for the donors in table 1. Up: quasi-static tests. Down: dynamic tests.

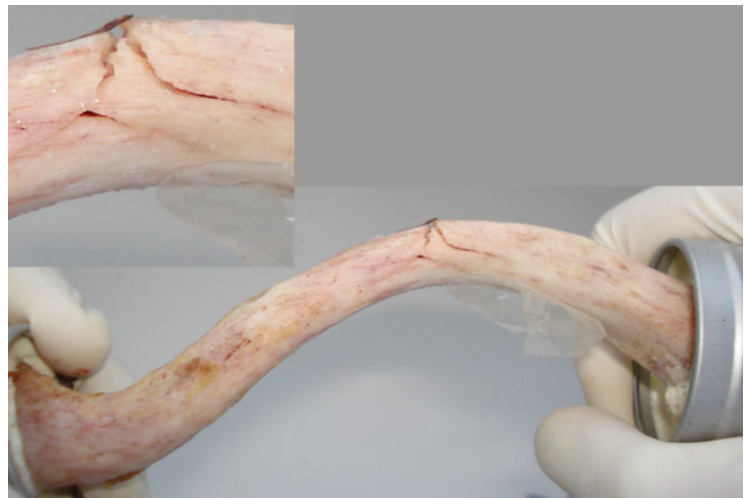

Figure 5: A typical butterfly-wing shape for a fracture, this is the most frequent type of fracture observed in the sample. 


\section{Discussion}

Only a modest amount of research has been conducted for the scientific analysis of fractures in clavicles. This fact sharply contrasts with the greater amount of research available for long bones. Specifically, in the case of lateral impact like a typical collision with a pedestrian or the falling of motorcyclist, the typical injury mechanism is axial compression. Duprey and coworkers conducted 18 dynamic compression tests with velocities $1.41 \pm 0.4 \mathrm{~m} / \mathrm{s}$ obtaining a typical fracture force value of $1470 \pm 460 \mathrm{~N}$ [8] (this value is about a $56 \%$ of the values found in this study) for compression tests. Maybe the higher ages used in the previous study can account for these discrepancies: there is a difference of 32 years in the PMHS in these studies $(76 \pm 12$ in Duprey's study and $44.8 \pm 12.4$ in the present study). Other researchers conducted quasi-static and dynamic tests $[11,12]$. Harnroongroj et al. performed 10 tests with velocity of $0.16 \mathrm{~mm} / \mathrm{s}$, obtaining a peak force value $1526 \pm 584 \mathrm{~N}$ [11]. This last value is about a $63 \%$ of the value obtained in the present study [the loading conditions in this last study were slightly different: a transversally force of $49 \mathrm{~N}$ was applied in the acromial and sternal extremities]. On the other hand, Zhang et al. conducted 10 dynamical tests with velocity of $100 \mathrm{~mm} / \mathrm{s}$ and obtained a fracture force of $2996 \pm 800 \mathrm{~N}$ [12]. This is a $24 \%$ of the value in the present study (the PMHSs used by Zhang were 44 older in average than those used in the present study). One of the important conclusions of our work is that the strength of a clavicle is significantly different in quasi-static and dynamic conditions ( $p$-values $<0.04$, Shapiro-Wilk test was used for normality, Fisher and Levene tests were used for variance equality and $t$-Student test for mean difference). For this reason, results from quasi-static tests should not be used to predict risks in situations such as fall injuries or traffic collisions, which are typically dynamic situations with high strain rates.

With respect to the location of the fractures, it has been reported that approximately $82 \%$ of the fractures occur in the middle third, coinciding with the pattern of injuries derived from the binding constraints [2]. Recently, another research group studied 68 clavicle fractures in the sternal third, where $69 \%$ are associated with traffic accidents and $9 \%$ with falls of more than one meter high[13]. This fact suggests that the location of the fractures is closer to the acromial third observed in the dynamic tests, as they are associated with highenergy crashes. The difference in the location of the fracture was also studied in [2], finding two incidence peaks, for younger than 30 years and for older than 80 years, but showing different patterns in fracture location: fracture in the middle third was most frequently associated with young people, while the limits between middle third and sternal/acromial thirds, i.e. borderline areas between thirds, were associated with older people.

The experimental data obtained in this study show that the clavicles exhibit a greater strength to fracture when the force is applied dynamically, which is a typical condition in road traffic collisions, since they are high energy events. The dynamic tests result in greater fracture forces than the static ones; in particular 
the $50 \%$ probability of fracture in this study is $2335 \mathrm{~N}$ for the quasi-static load case, and $3045 \mathrm{~N}$ for the dynamic load case. There are at least two possible interpretations, not mutually exclusive, that can explain this phenomenon, the failure models of brittle materials and the viscoelastic behavior of biological materials, including bone.

The Johnson-Holmquist failure model for brittle materials can provide a possible explanation of this higher stress for higher strain rates. This failure model has been shown to be adequate for materials that have a high compression strength, but that under traction tend to present micro-structural damage due to the growth of micro-fractures. Qualitatively, this situation is what happens in bone. By this reason, Johnson-Holmquist's criterion [14] is considered widely adequate for bone and it predicts that the fracture stress will be indicated by the following equation:

$$
\frac{\sigma_{f r a c}}{\sigma_{e}}=B[1+C \ln \dot{\varepsilon}]
$$

where $\sigma_{f r a c}$ is the stress of fracture, $\sigma_{e}$ is the yield stress, $B$ and $C$ are constants and $\dot{\varepsilon}$ is the strain rate. Such damage models predict that the higher the strain rate, the higher the fracture loads, as it is observed for clavicle fractures under uniaxial compression. It is interesting to observe that in models like the Johnson-Holmquist model, the viscoelastic effects combined with simpler damage models also lead to higher fracture stress. Some additional comments about this and the relevance of such models for the present study are presented in section ??.

Finally, with respect to the localization of fractures, several studies from hospitals have examined polytraumatic patients who have suffered a high-energy impact and a fractured clavicle and have shown high mortality, ranging from $20 \%$ to $34 \%$, associated primarily with thoracic and head trauma [16, 17]. This information points to the benefits of the training in scientific methodology, the creation of alliances with research support services, with multidisciplinary groups and belonging to different communities of researchers, guidelines recently indicated to the group of Emergency Physicians [18].

\section{Conclusions}

This study provides new information about the behavior of the clavicle under axial compression and under two loading conditions: quasi-static and dynamic (this later associated to a high energy impact). A total of 24 clavicles have been tested, 14 quasi-statically with a peak force of $2416 \pm 761 \mathrm{~N}$ and 10 dynamically with a peak force of $3183 \pm 981 \mathrm{~N}$.

The results of these tests show a greater stiffening of the clavicle as an effect of the increase of the impact speed, as can be the case in a road traffic crash. The present data quantify that the $50 \%$ probability of fracture is obtained at $2335 \mathrm{~N}$ for the quasi-static load case, and $3045 \mathrm{~N}$ for the dynamic load case.

Further work is needed in order to see if a wider range of confirmation rates could validate the hypothesis that the Johnson-Holmquist failure model is really 
adequate for explaining the higher observed strength under dynamic loading, when compared to quasi-static tests. This would improve our understanding of strength mechanisms and could help us decide whether the assumptions behind the Johnson-Holmquist model are really involved in the behavior of bone tissue. The obtained pattern of clavicle fracture is consistent with that found in road crashes with a greater frequency in the middle third. Indeed, the clavicle fracture in a distributed lateral impact is mainly associated to thoracic and cranioencephalic traumas. This study quantifies the force needed by the clavicle to fracture and consequently provides the explanation for the presence of associated injuries in anatomical regions near the upper extremity such as the thorax or the head.

Acknowledgements. The authors would like to thank the Centro para el Desarrollo Tecnológico Industrial (CDTI) for its funding of the CENIT project: Intelligent and Adaptive Lateral Protection Functions Technologies [reference CEN 20081031], research from which the present study is derived. Likewise, they would like to thank the team from the European Center of Injury Prevention in Navarre, specifically Eduardo del Pozo de Dios who conducted the tests. Also to Rafael Teijeira for coordinating the obtaining of the biological material, design of the study.

\section{Supplement: Additional Mathematical details}

In section 4, equation (3) was mentioned. In the same section it was stated that viscoelastic effects combined with simpler damage models also lead to higher fracture stress. In this section, we present a brief justification of this statement. For this purpose, the standard linear viscoelastic solid model will be considered. For a uniaxial compression test such as that considered in this study, the standard model of viscoelastic solid is governed by the following Boltzmann integral form $[15]$ :

$$
\sigma(t)=E_{0} \varepsilon(t)+E_{v} \int_{0}^{t} e^{-E_{v}(t-\tau) / \eta} \dot{\varepsilon}(\tau) \mathrm{d} \tau
$$

where $\sigma$ is the stress, $\varepsilon$ is the strain, and $\dot{\varepsilon}$ the strain rate. This model depends on three material parameters $E_{0}, E_{v}$ and $\eta$. Now, taking $\dot{\varepsilon}$ as constant, $t=\varepsilon / \dot{\varepsilon}$ and integrating, we obtain explicitly:

$$
\sigma=E_{0} \varepsilon+E_{v} \frac{\dot{\varepsilon}}{\omega}\left(1-e^{-\frac{\omega \varepsilon}{\dot{\varepsilon}}}\right)=\sigma_{e}(\varepsilon)+\sigma_{v}(\varepsilon, \dot{\varepsilon})
$$

In this equation we have introduced the constant value $\omega=E_{v} / \eta$ which is the time constant associated with the viscoelastic delay. The $\sigma$ is the stress which has been divided into an elastic $\sigma_{e}(\varepsilon)=E_{0} \varepsilon$ contribution and a viscoelastic contribution $\sigma_{v}=\sigma-\sigma_{e}$. Equation (5) shows that in dynamic tests, a higher strain will always be present given a stress level, just as in our experimental data (this implies that $\mathrm{d} \sigma_{v} / \mathrm{d} \varepsilon>0$ which will be useful in the following). If we 
assume that failure of the bone tissue has a maximum toughness, and a level of stress $\sigma_{0}$, then the toughness is given by:

$$
U_{\tau}=\int_{0}^{\varepsilon_{0}} \sigma(\varepsilon) \mathrm{d} \varepsilon, \quad \text { with } \sigma_{0}=E_{0} \varepsilon_{0}+\sigma_{v}\left(\varepsilon_{0}, \dot{\varepsilon}\right)
$$

If we define a function $\phi_{\dot{\varepsilon}}^{-1}\left(\varepsilon_{0}\right)=E_{0} \varepsilon_{0}+\sigma_{v}\left(\varepsilon_{0}, \dot{\varepsilon}\right)$, then the condition $\mathrm{d} \sigma_{v} / \mathrm{d} \varepsilon>$ 0 , deduced from (5) for any $\dot{\varepsilon} \geq 0$, ensures that $\phi_{\dot{\varepsilon}}^{-1}\left(\varepsilon_{0}\right)$ is a monotonically increasing function [in addition, it is surjection in $\mathbb{R}^{+}$] which implies that the inverse $\phi_{\dot{\varepsilon}}\left(\sigma_{0}\right)$ exists and will be a monotonically increasing function of $\sigma_{0}$. In this way, the assumption of a criterion failure based on maximum toughness explains that for a higher strain rates, the failure occurs at higher stress. The Leibniz integral rule applied to equation (6) lead to the expression:

$$
\frac{\partial U_{\tau}}{\partial \dot{\varepsilon}}=\sigma\left(\varepsilon_{0}\right) \frac{\partial \phi_{\dot{\varepsilon}}\left(\varepsilon_{0}\right)}{\partial \dot{\varepsilon}}<0
$$

This happens because the derivative is always negative as the sign of $\partial \phi_{\dot{\varepsilon}} / \partial \dot{\varepsilon}$ coincides with the sign of $-\partial \sigma_{v} / \partial \dot{\varepsilon}<0$. This heuristic argument suggests another reason for which we found a higher strength for high rate situations: being the toughness (work to fracture) higher for quasi-static conditions, for the same level of stress or force the dynamic situations imply a smaller work to fracture due to a much lower strain/displacement.

\section{References}

[1] Franmpton RJ, Morris AP, Thomas P, Bodiwala GG. An overview of upper extremity injuries to car occupants in UK vehicles crashes, Proc IRCOBI Conference 37-52, 1997.

[2] Khan LAK, Bradnock TJ, Scott C, Robinson MC. Fractures of the Clavicle, J Bone Joint Surg Am 91:447, 2009.

[3] Fernández-Guerrero IM, Burbano Santos P, Martín-Sánchez FJ, HidalgoRodríguez A, Leal-Lobato MM, Riula-Doce C. Producción científica de los urgenciólogos españoles durante el quinquenio 2010-2014 y su comparación con el quinquenio 2005-2009, Emergencias 28:153, 2016.

[4] Postacchini R, Gumina S, Farsetti P, Postacchini F. Long-term results of conservative management of midshaft clavicle fracture, International orthopaedics, 34(5):731, 2010.

[5] Robinson CM. Fractures of the clavicle in the adult. Epidemiology and classification, J Bone Joint Surg [Br] 80:467-484, 1998.

[6] Liu GD, Tong SL, Ou S, Zhou LS, Fei J, Nan GX, Gu JW. Operative versus non-operative treatment for clavicle fracture: a meta-analysis, International orthopaedics, 37(8):1495, 2013. 
[7] Compigne S, Caire Y, Quesnel T, Verriest JP. Non-injurious and injurious impact response of the human shoulder - Three dimensional analysis of kinematics and determination of injury threshold, Stapp Car Crash Journal 48:89, 2004.

[8] Duprey S. Modélisation en elements finis du complexe de l'epaule et simulation de sa réponse à un choc lateral. Dissertation, Institut national des sciences appliquées de Lyon. France, 2007.

[9] Duprey S, Bruyère K, Verriest JP, Influence of geometrical personalization on the simulation of clavicle fractures, Journal of Biomechanics 41:200, 2008 .

[10] Arregui-Dalmases C, Del Pozo E , Duprey S , Lopez-Valdes F, Anthony L, Subit D. A Parametric Study of Hard Tissue Injury Prediction Using Finite Elements: Consideration of Geometric Complexity, Subfailure Material Properties, CT Thresholding, and Element Characteristics, Traffic Injury Prevention 11(3):286, 2010.

[11] Harnroongroj T., Tantikul C, Keatkor S. The clavicular fracture: A biomechanical study of the mechanism of clavicular fracture and modes of fracture, J Med Assoc Thai 83:663, 2000.

[12] Zhang Q, Kindig MW, Li ZP, Crandall JR, Kerrigan JR. Development of structural and material clavicle response corridors under axial compression and three point bending loading for clavicle finite element model validation, Journal of Biomechanics 47(11):2563, 2014.

[13] Salipas A, Kimmel LA, Edwards ER, Rakhra S, Moaveni AK. Natural history of medial clavicle fractures, Injury 47(10):2235, 2016.

[14] Johnson GR, Holmquist, TJ. An improved computational constitutive model for brittle materials, AIP Conference Proceeding 309:981, 1994.

[15] Lakes R. S. Viscoelastic materials. Cambridge University Press ISBN 978 052188568 3, 2009.

[16] McKee MD, Schemitsch EH, Stephen DJ, Kreder HJ, Yoo D, Harrington J. Functional outcome following clavicle fractures in polytrauma patients, J Trauma 47(3):616, 1999.

[17] Taitsman L, Nork SE, Coles CP. Open clavicle fractures and associated injuries, J Orthop Trauma 20(6):396, 2006.

[18] Llorens P. Urgenciólogo e investigador: una combinación posible e imprescindible, Emergencias 28:143, 2016. 